\title{
Kaempferol Protects Renal Fibrosis through Activating the BMP-7- Smad1/5 Signaling Pathway
}

\author{
Xiaojun Ji, ${ }^{a}$ Jing Cao, ${ }^{b}$ Liting Zhang, ${ }^{a}$ Zhirui Zhang, ${ }^{a}$ Weiwei Shuai, ${ }^{b}$ and Wu Yin*,a \\ ${ }^{a}$ State Key Lab of Pharmaceutical Biotechnology, College of Life Sciences, Nanjing University; Nanjing 210046, \\ China: and ${ }^{b}$ Department of Pharmacy, Women's Hospital of Nanjing Medical University (Nanjing Maternity and \\ Child Health Care Hospital); Nanjing 210004, China.
}

Received November 14, 2019; accepted December 21, 2019

\begin{abstract}
Renal interstitial fibrosis (RIF) is a common pathological characteristic associated with end-stage renal disease. However, treatment strategies for RIF are still very limited. In this study, we reported that kaempferol, a classic flavonoid, exhibited strong and widely inhibitory effect on the expression of fibrosis related genes in transforming growth factor beta 1 (TGF- $\beta 1)$ treated NRK-52E cells. Further studies revealed that kaempferol inhibited TGF- $\beta 1$ induced epithelial-mesenchymal transition (EMT) process of NRK-52E cells and improved renal function deterioration and RIF in unilateral ureteral obstruction (UUO) rats. After exploring the underlying mechanisms, we found that kaempferol was able to activate the BMP-7-Smad1/5 pathway, rather than the TGF- $\beta 1-S m a d 2 / 3$ pathway. To further validate these results, DMH1 and BMP-7 knockdown were utilized at the cellular level and the results showed that both methods were able to antagonize the effects of kaempferol on the EMT process of NRK-52E cells induced by TGF- $\beta 1$. In UUO rats, inhibition of BMP-7 signaling by DMH1 also reversed the effects of kaempferol on renal function decline and RIF. Taken together, our findings demonstrated that kaempferol could be a good candidate for renal fibrosis treatment.
\end{abstract}

Key words kaempferol; renal interstitial fibrosis; epithelial-mesenchymal transition; BMP-7; Smad1/5

\section{INTRODUCTION}

Renal fibrosis is considered to be one of the characteristics of progressive renal injury and is a common feature of end-stage chronic kidney diseases. ${ }^{1)}$ Renal fibrosis is mainly classified into glomerular sclerosis and renal interstitial fibrosis (RIF), of which RIF is the most widely studied. ${ }^{2)}$ Transforming growth factor beta (TGF- $\beta$ ) is a key regulator of renal fibrosis, its role in renal fibrosis has been extensively studied. ${ }^{3-6)}$ It is pointed out that that TGF- $\beta 1$ mediates renal fibrosis through the increase of extracellular matrix (ECM) production and the reduction of ECM degradation. ${ }^{5}$ In addition, TGF- $\beta 1$ was able to promote epithelial-mesenchymal transition (EMT) of renal tubular epithelial cells. ${ }^{78}$ Pirfenidone (PFD) is an active compound targets TGF- $\beta$ signaling by reducing the promoter activity of $T g f b l$ and it was reported to alleviate RIF and glomerular sclerosis induced by diabetes. ${ }^{9,10}$

In recent years, BMP-7, another protein in TGF- $\beta$ superfamily, has attracted attention due to its effect in preventing the role of TGF- $\beta 1$ in fibrosis. BMP-7 is a homodimeric protein and is a naturally-derived negative regulator of TGF- $\beta / \mathrm{Smad}$ signaling pathway. ${ }^{11)}$ BMP-7 is highly expressed in normal kidneys, while it was reported to be downregulated in various kidney diseases such as diabetic nephropathy and hypertensive nephrosclerosis. ${ }^{12,13)}$ BMP-7 was capable of alleviating RIF in unilateral ureteral obstruction (UUO) rats and diabetic rats. ${ }^{14,15)}$ In addition to improving renal fibrosis, BMP-7 treatment attenuated EMT process of renal tubular epithelial cells induced by TGF- $\beta 1$ and reduced ECM production in mesangial cells. ${ }^{16-18)}$

Our previous study has shown that Ginkgo biloba extract (GbE) attenuated RIF caused by diabetes. GbE is a multicomponent mixture contains flavonoids and terpene lactones.
The flavonoids that have been identified mainly include rutin, myricetin, quercetin, luteolin, kaempferol, isorhamnetin and genistein. ${ }^{19)}$ Among the flavonoid components, the effects of rutin, quercetin and myricetin have been reported in renal fibrosis induced by diabetes and UUO and in EMT process of renal tubular epithelial cells. ${ }^{20-23)}$ However, the effects of luteolin, kaempferol, isorhamnetin and genistein on RIF are rarely studied. This study aimed to evaluate the effects of these four compounds on renal fibrosis in vitro and in vivo.

\section{MATERIALS AND METHODS} S1.

Reagents Reagents used in this study are listed in Table

Cell Culture and Treatment The rat proximal tubular epithelial cell line NRK-52E was a kind gift from Xuzhou Medical University (Xuzhou, Jiangsu, China). Cells were cultured in Dulbecco's modified Eagle's medium (DMEM) (with 10\% fetal bovine serum (FBS)). The cells were serum starved for $24 \mathrm{~h}$ prior to treatment. To induce EMT, the cells were treated with TGF- $\beta 1(5 \mathrm{ng} / \mathrm{mL})$ for $24 \mathrm{~h}$. Genistein, isorhamnetin, kaempferol and luteolin were dissolved in $0.1 \%$ dimethyl sulfoxide (DMSO) to $0.5,1,5,10,20,40,80,100$, and $200 \mathrm{mM}$, respectively, and added to the medium at a ratio of $1: 1000(\mathrm{v} / \mathrm{v})$. To inhibit the TGF- $\beta 1$ or BMP-7 signaling pathways, the cells were treated with LY3200882 $(1 \mu \mathrm{M})$ or DMH1 $(20 \mu \mathrm{M})$, respectively.

Animals and Study Design Male Sprague-Dawley rats, weighing $180-220 \mathrm{~g}$, were purchased from the Laboratory Animal Center of Xuzhou Medical University. The performance of UUO and Sham rats were described previously. ${ }^{24)}$ For the first animal experiment, the UUO rats were randomly divided into 5 groups with 8 animals in each 
group, namely an UUO group (UUO), a low-dose kaempferol group (UUO $+\mathrm{KL}, 30 \mathrm{mg} / \mathrm{kg} / \mathrm{d}$ ), a medium-dose kaempferol group (UUO + KM, $60 \mathrm{mg} / \mathrm{kg} / \mathrm{d}$ ), a high-dose kaempferol group (UUO $+\mathrm{KH}, 90 \mathrm{mg} / \mathrm{kg} / \mathrm{d}$ ) and a pirfenidone group $(\mathrm{UUO}+\mathrm{PFD}, 500 \mathrm{mg} / \mathrm{kg} / \mathrm{d})$. For the second animal experiment, the UUO rats were randomly divided into 3 groups with 8 rats in each group, namely a UUO group (UUO), a medium-dose kaempferol group (UUO $+\mathrm{KM}, 60 \mathrm{mg} / \mathrm{kg} / \mathrm{d}$ ) and a medium-dose kaempferol group supplemented with DMH1 $(\mathrm{UUO}+\mathrm{KM}+\mathrm{DMH} 1,40 \mathrm{mg} / \mathrm{kg} / \mathrm{d})$. In the two experiments, rats in the sham group ( $n=8$ rats) were set as the control. Kaempferol, pirfenidone and DMH1 were dissolved in $1 \%$ CMC-Na and treated the rats at a volume of $0.5 \mathrm{~mL}$ per $100 \mathrm{~g}$ body weight in an intragastric manner. Rats in the sham and UUO groups were received the same volume of $1 \% \mathrm{CMC}-\mathrm{Na}$. After treatment for $14 \mathrm{~d}$, blood and kidney samples of the rats were collected. All the animal experiments were approved by the Animal Ethics Committee of Xuzhou Medical College and were conducted in accordance with the Guide for the Care and Use of Laboratory Animals provided by the National Institutes of Health (Bethesda, MD, U.S.A.).

Renal Function Assessment The relative kidney body weight $(\mathrm{mg} / \mathrm{g})$ was determined as the ratio of the left or the right kidney and the body weight of the rat. Creatinine $(\mathrm{Cr})$ and blood urea nitrogen (BUN) levels were measured using $\mathrm{Cr}$ and BUN assay kits according to the manufacturer's instructions.

CCK-8 Analysis NRK-52E cells were cultured in 96-well microplates at a density of 5000 cells/well. Twenty-four hours after cultivation, the cells were serum starved and treated with different concentrations of genistein, isorhamnetin, kaempferol and luteolin for 12, 24, and $48 \mathrm{~h}$. Cytotoxicity was performed using the CCK-8 assay following the manufacturer's instruction.

RNA Interference NRK-52E cells were cultured in 6 -well plates at a density of $5 \times 10^{5}$ cells/well. When the cell confluent reached $60-70 \%$, cells were changed with the OptiMEM medium. The scramble or BMP-7 siRNAs (100 pmols) were transfected into NRK-52E cells using lipofectamine 2000 transfection reagent according to the manufacturer's instruction. The scramble and BMP-7 siRNAs were synthesized at GenePharma (Shanghai, China). The sequences are shown in Table S2.

Western Blotting Analysis Total protein from NRK$52 \mathrm{E}$ cells and the kidney tissues was extracted with RIPA lysis buffer. For immunoblotting, an equal amount of protein $(40 \mu \mathrm{g})$ was separated on $8-12 \%$ sodium dodecyl sulfate-polyacrylamide gel electrophoresis (SDS-PAGE) and transferred onto polyvinylidene difluoride (PVDF) membranes. After transferring, the membranes were blocked in 5\% nonfat milk, followed by incubation with primary antibodies overnight at $4^{\circ} \mathrm{C}$. The dilution ratios $(\mathrm{v} / \mathrm{v})$ of the primary antibodies are as follows: E-cadherin $(1: 1000), \alpha$-smooth muscle actin $(\alpha$-SMA) $(1: 2000)$, Collagen I $(1: 2000)$, Collagen III $(1: 1000)$, pSmad1/5 (1:1000), p-Smad2/3 (1:1000), Smad1 (1:1000), Smad5 (1:1000), Smad2/3 (1:1000), BMP-7 (1:1000) and $\alpha$-Tubulin $(1: 5000)$. Blots were developed using HRP-conjugated secondary antibodies $(1: 50000)$. The quantification was performed through the intensity measurement using the ImageJ software (Version 1.52a, NIH, MD, U.S.A.).

RNA Isolation and Quantitative (q)RT-PCR Total RNA was extracted from cells or frozen kidney tissues using a TRIzol reagent according to the manufacturer's instruction. The cDNA was synthesized using $0.5-1 \mu \mathrm{g}$ of total RNA and reverse transcribed using the qPCR RT kit. Real-time qRTPCR was used to detect the expression of fibrosis related genes. The method was as previously described. ${ }^{22)}$ Primers were synthesized at Sangon Biotech (Shanghai, China) and are listed in Table S3.

Histological Analysis Kidney samples were fixed in $4 \%$ paraformaldehyde and embedded in paraffin. Sections of $4 \mu \mathrm{m}$ thickness were cut for histological analysis. The sections were stained with the Masson's trichrome staining kit or Sirus Red staining kit according to the manufacturer's instructions, respectively. Then, the sections were observed and the pictures were taken using a BX43F microscope (Olympus, Tokyo, Japan). Percentage of the positive areas was determined by the Image Pro Plus Software (Version 6.0, Media Cybernetics, MD, U.S.A.).

Immunohistochemistry Analysis Paraffin embedded kidney sections were deparaffinized in xylene and hydrated in graded alcohol and water, then were treated with $3 \% \mathrm{H}_{2} \mathrm{O}_{2}$. After blocking with 5\% BSA, the sections were incubated with the primary antibody collagen I $(1: 200)$ at $4^{\circ} \mathrm{C}$ overnight. Then the sections were incubated with HRP-labelled secondary antibody $(1: 1000)$ at room temperature. A DAB reagent was used to perform visualization. After hematoxylin counterstaining, differentiation, dehydration and transparency, the sections were examined using an Olympus BX43F microscope. The protein expression was quantified by the Image Pro Plus Software.

Statistical Analysis Data were presented as the mean \pm standard deviation (S.D.) All data were analyzed using the SPSS software (Version 24.0, IBM, NY, U.S.A.). For multiple comparisons, ANOVA was applied with LSD post hoc analysis for data meeting homogeneity of variance or with Dunnett's T3 analysis for data not assuming equal variances. In all cases, differences were considered significant at $p<0.05$.

\section{RESULTS}

Kaempferol Inhibits EMT of NRK-52E Cells Induced by TGF- $\boldsymbol{\beta} 1$ We first measured the effects of genistein, isorhamnetin, kaempferol and luteolin on the viability of NRK-52E cells. The results showed that the four compounds had little effect on the viability of NRK-52E cells when the concentrations are lower than $40 \mu \mathrm{M}$ (Fig. 1a and Figs. S1a-c). Therefore, three concentrations of the four compounds $(5,10$ and $20 \mu \mathrm{M}$ ) were selected for subsequent experiments. Cells were treated with TGF- $\beta 1$ to induce EMT and PFD was set as a positive control. The results showed that high dose of genistein slightly downregulated the expression of Colla2 and Ctgf genes induced by TGF- $\beta 1$ (Fig. S1d). Treatment with isorhamnetin at a high dose was able to inhibit the gene expression of Colla1, Colla2, Ctgf and Fnl (Fig. S1e). Likewise, luteolin showed to downregulate the gene expression of Colla 2 and Col3al (Fig. S1f). However, kaempferol showed a generally strong inhibitory effect on expression of these fibrotic genes at medium and high doses (Fig. 1b). Therefore, we focused on the role of kaempferol in RIF in the later experiments.

Morphological changes of the cells further confirmed the 
a
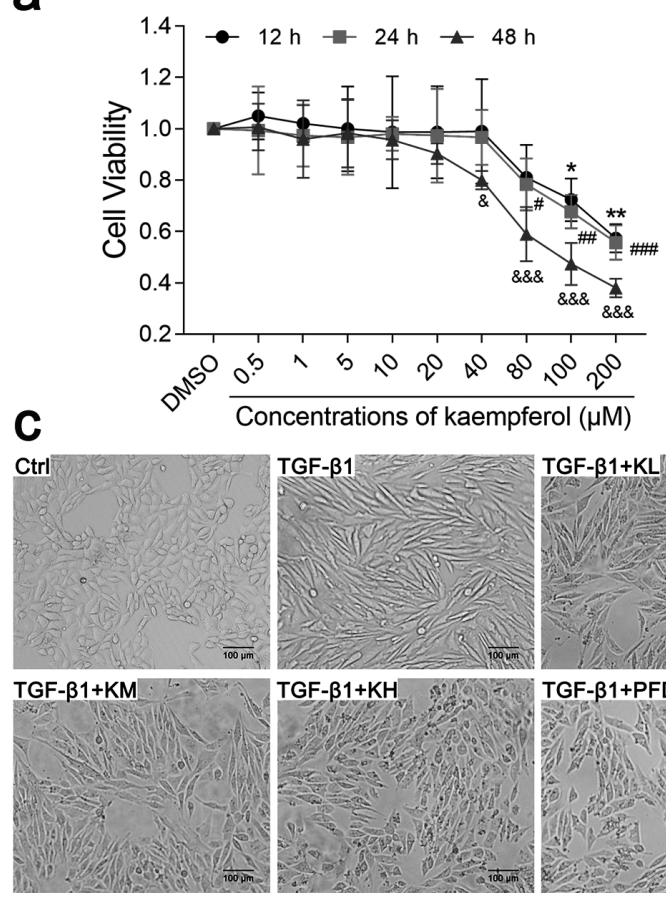

b
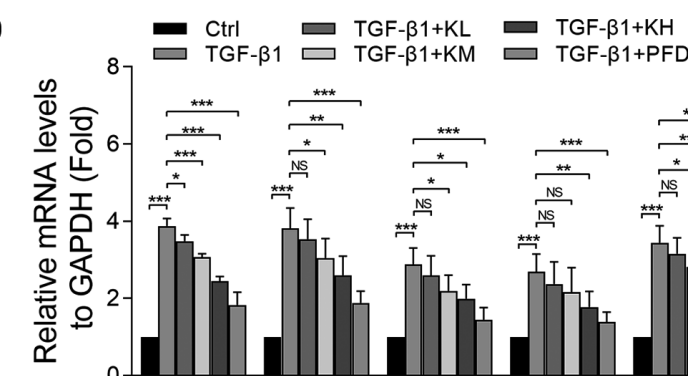
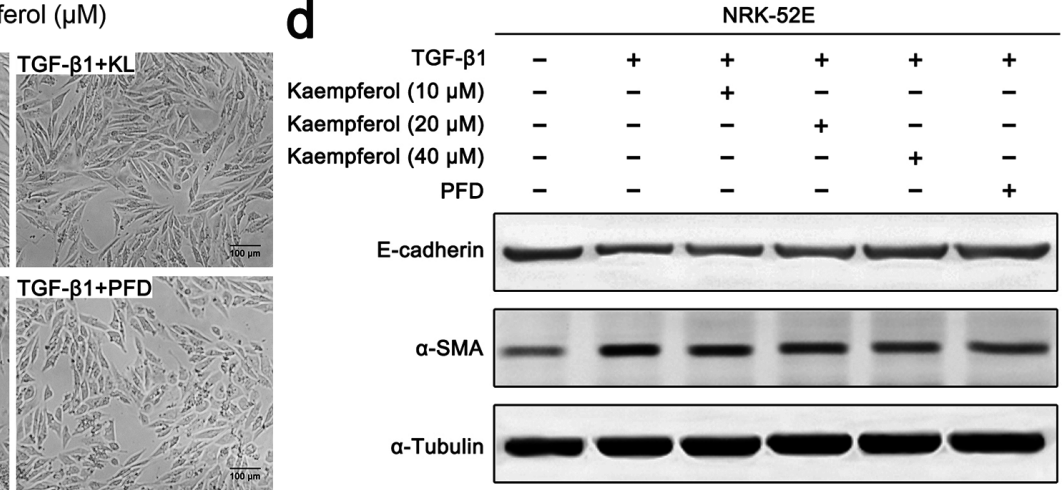

Fig. 1. Kaempferol Ameliorates the EMT Process of NRK-52E Cells Induced by TGF- $\beta 1$

(a) Viability of the cells treated with kaempferol was measured by CCK-8 assay. (b) Gene expression of Collal, Colla2, Col3al, Ctgf, Fn1 and Acta2 in cells was measured by qRT-PCR. (c) Cell morphology was observed by an inverted microscope. (d) Protein expression of E-cadherin, $\alpha$-SMA was detected by Western blotting. All

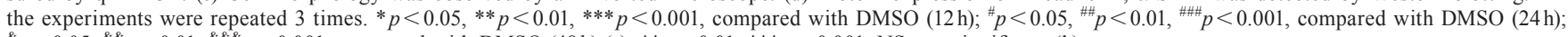
${ }^{\star} p<0.05,{ }^{\& \&} p<0.01,{ }^{\& \&} p<0.001$, compared with DMSO (48h) (a). ${ }^{* *} p<0.01 ;{ }^{* * *} p<0.001$; NS, not significant (b).

results above. The long fusiform morphology induced by TGF- $\beta 1$ was reversed after kaempferol treatment (Fig. 1c). Moreover, the expression of epithelial phenotype E-cadherin in the cells was downregulated after TGF- $\beta 1$ treatment. In contrast, the mesenchymal protein $\alpha$-SMA was upregulated. Medium and high doses of kaempferol treatment showed a strong reversal effect on the protein expression of E-cadherin and $\alpha$-SMA induced by TGF- $\beta 1$ (Fig. $1 d$ and Fig. S2).

Kaempferol Improves RIF in Rats Induced by UUO To study the effect of kaempferol on RIF, we prepared a UUO rat renal fibrosis model. The results showed that the left ureteral obstruction induced an enlargement of both the left and right kidneys of the rats. Kaempferol and PFD treatment reversed this phenomenon to varying degrees (Figs. 2a, b). In addition, kaempferol dose-dependently improved renal function decline in UUO rats (Figs. 2c, d). In addition, we examined the effects of kaempferol on expression of fibrosis related proteins and genes in the kidneys of UUO rats. Western blotting showed that Kaempferol in different doses reversed the downregulation of E-cadherin, and the effect of high dose kaempferol was better than PFD (Fig. 2e and Fig. S3a). Consistently, kaempferol was able to prevent the accumulation of $\alpha$-SMA in the kidneys of UUO rats (Fig. 2e and Fig. S3b). Besides, kaempferol exerted strong inhibitory effects on abnormal expression of Collagen I and Collagen III induced by UUO. (Fig. 2e and Figs. S3c, d). qRT-PCR showed that medium and high doses of kaempferol exerted inhibitory effect on the expression of fibrosis related genes dose-dependently, of which the effect of high dose of kaempferol was close to that of PFD (Fig. 2f).

In order to evaluate the degree of renal fibrosis more directly, we applied Masson's trichrome staining to observe the amount and distribution of collagens in the kidney. Masson- positive areas in the kidneys of UUO rats treated with kaempferol or PFD reduced obviously (Fig. $2 g$ and Fig. S3e). The results in Sirus Red staining were consistent with that in the Masson staining (Figs. S3g, h). In addition, we also detected the expression of Collagen I in kidney sections of the rats using immunohistochemistry. In accordance with the above results, a large amount of Collagen I was found deposited in the kidney of UUO rats, mainly in the renal interstitium. Both medium and high doses of kaempferol downregulated the content of this protein (Fig. $2 \mathrm{~h}$ and Fig. S3f).

Kaempferol Prevents EMT of NRK-52E Cells Induced by TGF- $\beta 1$ through the BMP-7-Smad1/5 Pathway The TGF- $\beta 1-S m a d 2 / 3$ and BMP-7-Smad1/5 are two key pathways in fibrosis regulation. In this study, TGF- $\beta 1$ significantly induced the phosphorylation of Smad2/3, while inhibited the phosphorylation of Smad1/5. Kaempferol treatment restored the phosphorylation levels of Smad1/5 but had little effect on the activity of Smad2/3. LY3200882 and DMH1 verified the reliability of our results (Fig. 3a and Figs. S4a, b). Besides, kaempferol itself was able to upregulate the protein expression of BMP-7 and the phosphorylation of Smad1/5 slightly. However, it showed little effect on the phosphorylation levels of Smad2/3 (Fig. S4c). These results suggested that the effects of kaempferol are likely to be associated with its regulation on BMP-7 signaling pathway. To test this hypothesis, we first studied the effects of kaempferol on EMT process of NRK$52 \mathrm{E}$ cells induced by TGF- $\beta 1$ when treated with DMH1. The results showed that DMH1 reversed the effect of kaempferol on TGF- $\beta 1$ induced cell morphological changes and expression of fibrosis related genes (Fig. $3 b$ and Fig. S4d). In addition, DMH1 treatment notably reversed the phenomena that kaempferol prevented the down-regulation of E-cadherin and 
a
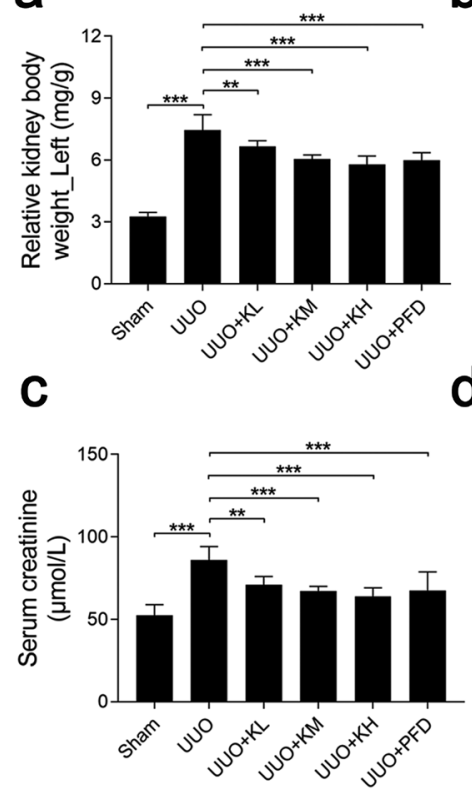

$g_{\mathrm{s}}$

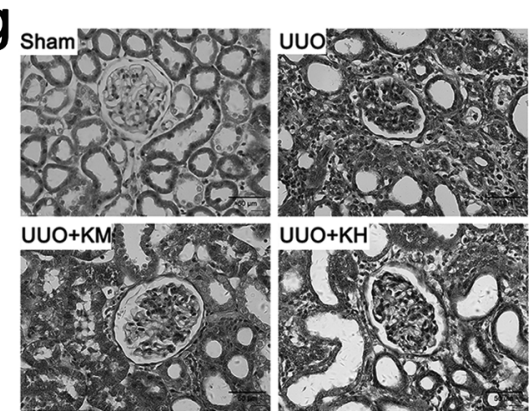

b

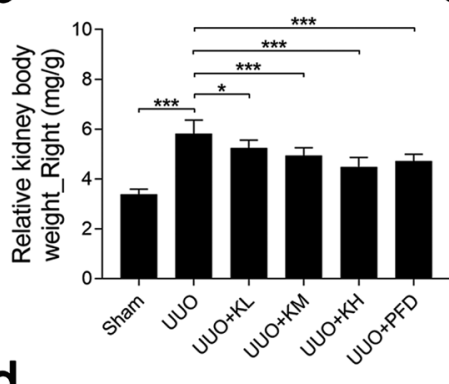

e
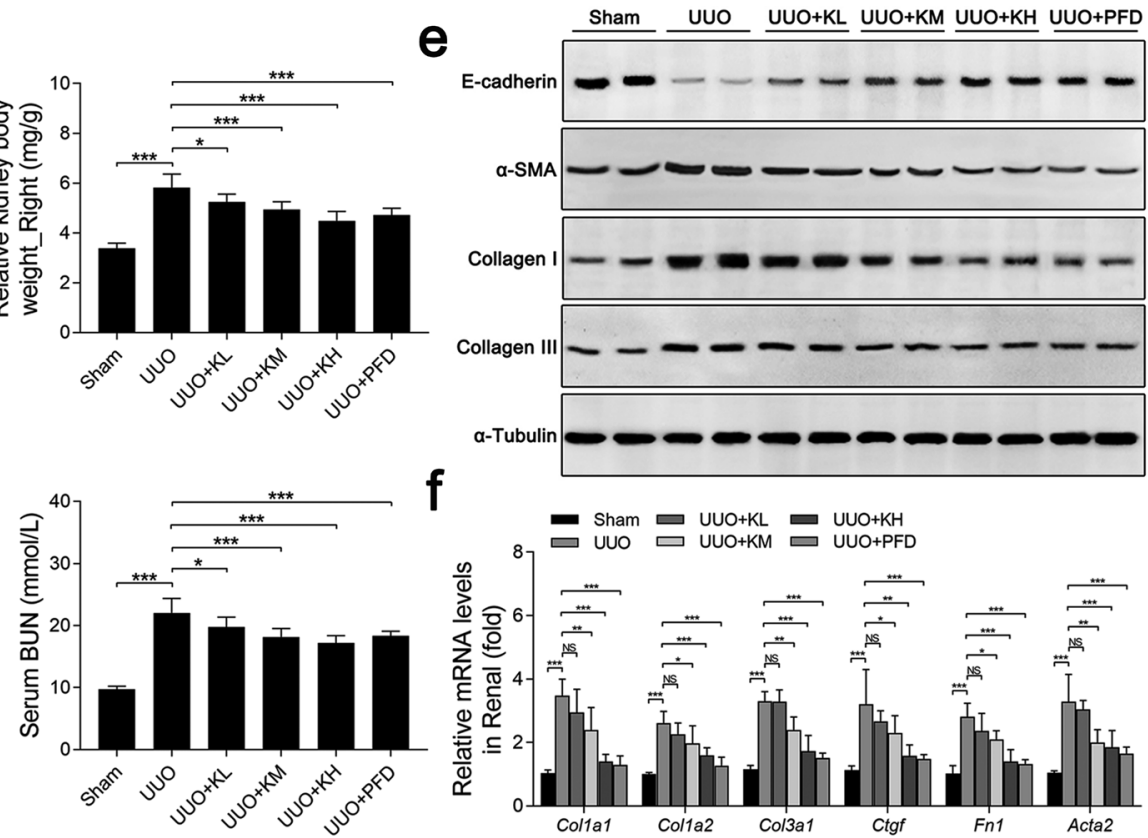

$f$

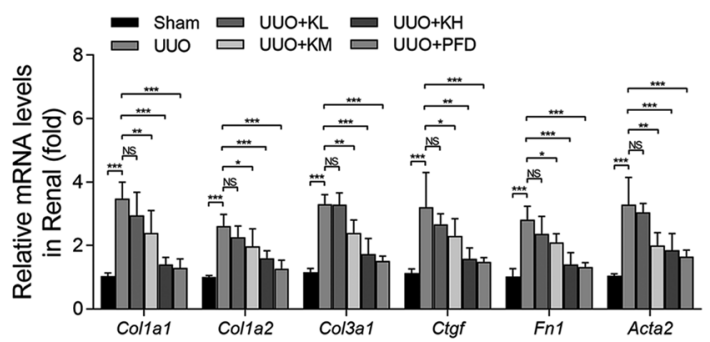

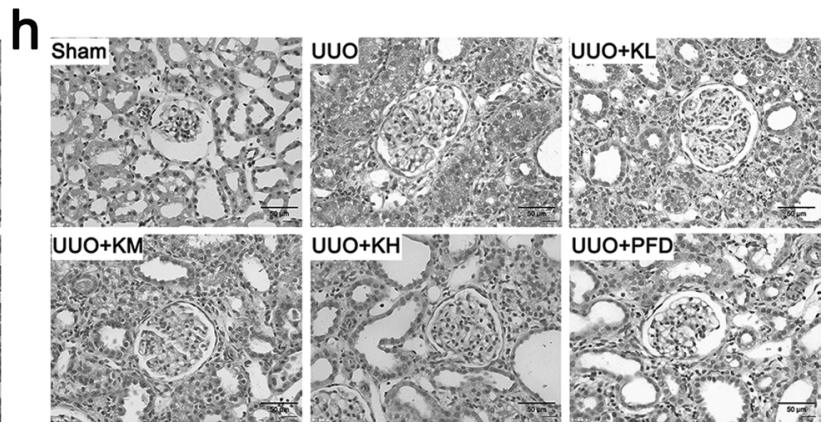

Fig. 2. Kaempferol Alleviates Renal Function Decline and Renal Interstitial Fibrosis in the UUO Rats

Relative weight of left (a) and right (b) kidneys to body weight of the rats was determined $(n=8)$. Serum creatinine (c) and BUN (d) levels of the rats were measured $(n=8)$. (e) Protein expression of E-cadherin, $\alpha$-SMA, Collagen I and Collagen III was detected by Western blotting ( $n=4)$. (f) Representative gene expression of Collal, Colla2, Col3al, Ctgf, Fnl and Acta2 in kidney of the rats was measured by qRT-PCR $(n=4)$. $(\mathrm{g})$ Masson staining of renal cortex sections of the rats in each group $(n=4)$. (h) Protein expression of Collagen I in renal cortex of the rats in each group through immunohistochemistry $(n=4){ }^{*} p<0.05$; $* * p<0.01 ; * * *<0.001$; NS, not significant.

the up-regulation of $\alpha$-SMA caused by TGF- $\beta 1$ (Fig. 3c and Figs. S4e, f).

Considering the fact that kaempferol can up-regulate the protein expression of BMP-7 (Fig. 3c and Figs. S4g, h), we studied the roles of BMP-7 interference on protective effects of kaempferol in vitro. It showed that BMP-7 knockdown blocked the improvement of kaempferol on cell morphological changes and gene expression of Colla1, Colla2, Col3al, Ctgf and $F n 1$ induced by TGF- $\beta 1$ (Fig. $3 \mathrm{~d}$ and Fig. S4i). In accordance with the role of DMH1, BMP-7 knockdown blocked kaempferol's effects on E-cadherin upregulation and $\alpha$-SMA downregulation (Fig. 3e and Figs. S4j-m).

Protective Effects of Kaempferol on Renal Fibrosis of Rats Induced by UUO Are Dependent on the BMP-7-Smad1/5 Pathway To further investigate the role of BMP-7 pathway in the protective effects of kaempferol on renal fibrosis in vivo, UUO rats were treated with DMH1. The results showed that DMH1 treatment not only antagonized the improvement of kaempferol on kidney hypertrophy and renal function decline in UUO rats (Figs. 4a-d), but also reversed the effect of kaempferol on protein expression of E-cadherin and $\alpha$-SMA (Fig. 4e and Figs. S5a, b). It is worth mentioning that the combined treatment with DMH1 and kaempferol totally restored the expression Collagen I and Collagen III, which was downregulated after kaempferol treatment (Fig. 4e and Figs. S5c-f). Moreover, administration of DMH1 combined with kaempferol up-regulated the gene expression of Collal, Colla2, Col3al, Ctgf and Fnl again compared with the rats treated with kaempferol alone (Fig. 4f). Masson staining revealed that the deposition of collagens in the kidney of UUO rats was notably reduced when treated with a medium dose of kaempferol. However, inhibition of BMP-7 signaling by DMH1 made this amelioration abolished (Fig. 4g and Fig. S5g). The results in Sirus Red staining and Collagen I immunohistochemistry staining were consistent with that in Masson staining (Figs. S5h-k).

\section{DISCUSSION}

RIF is one of the main features of progressive renal injury, in which EMT of the renal tubular epithelial cells is the central event. ${ }^{25)}$ The myofibroblasts can break through the basement membrane and transfer to the renal interstitium, secreting a large amount of ECM and eventually leading to renal function decline. ${ }^{25}$ ) There are various animal models of RIF, including UUO, 5/6 nephrectomy, drug induced nephrotoxic- 
a

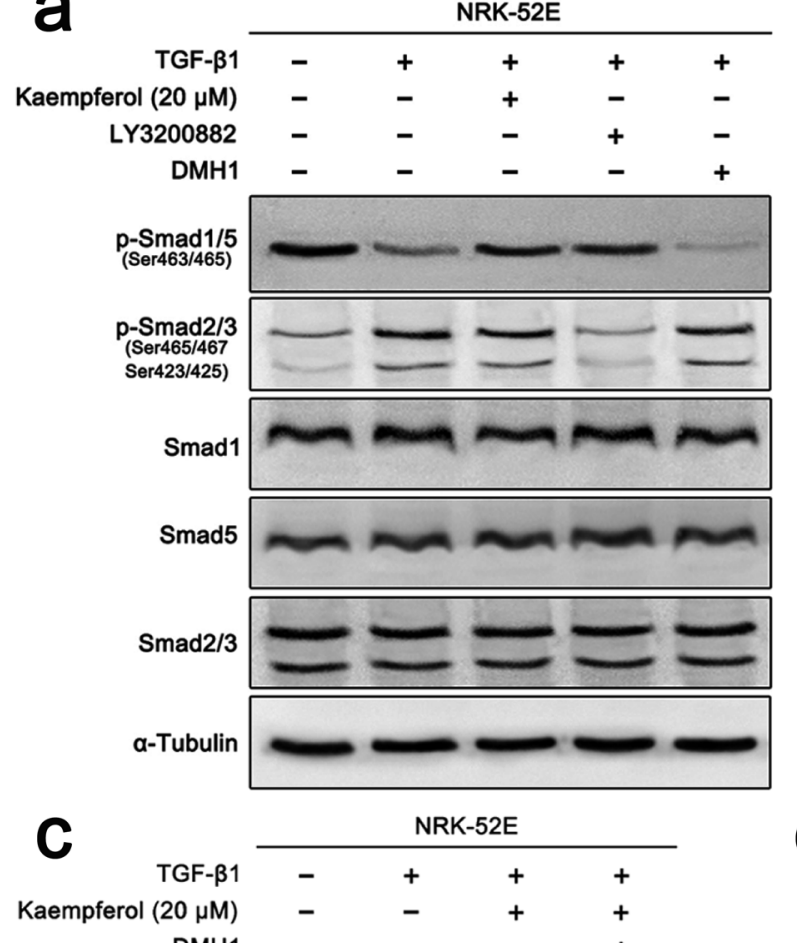

b
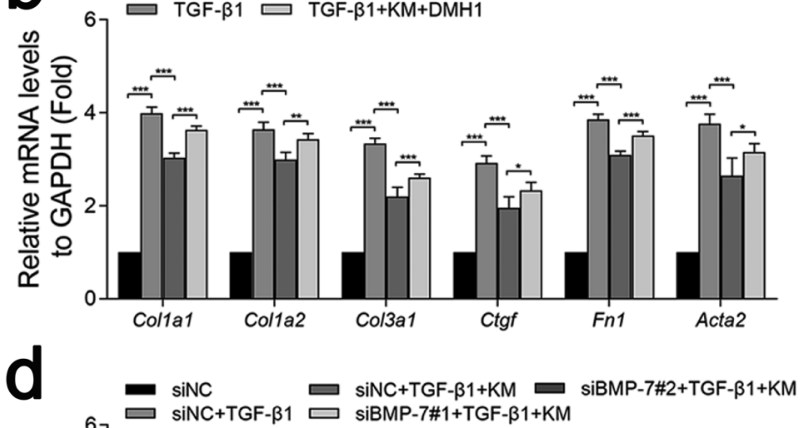
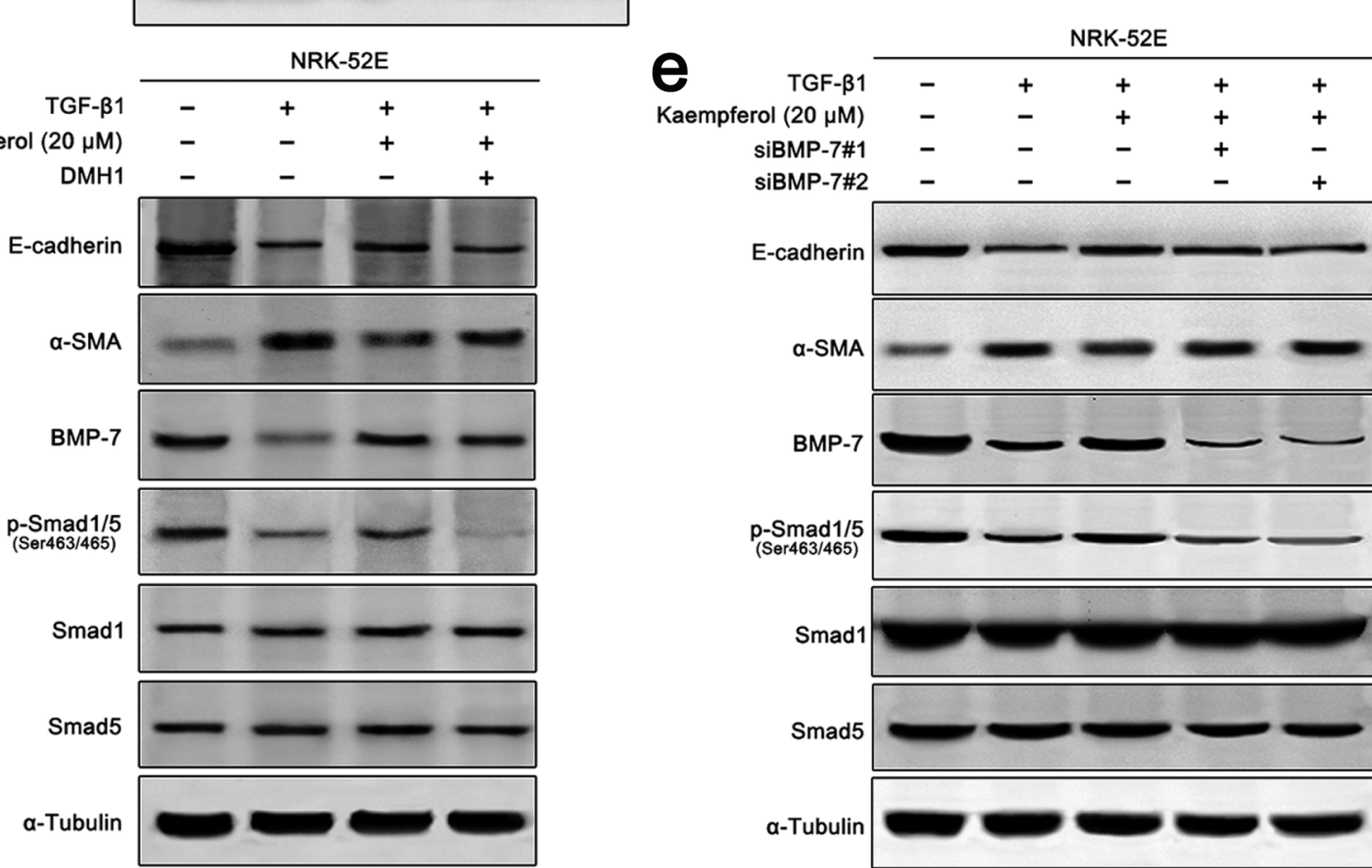

Fig. 3. Kaempferol Ameliorates the EMT Process of NRK-52E Cells Induced by TGF- $\beta 1$ through the BMP-7-Smad1/5 Pathway

(a) Protein expression of p-Smad1/5 (Ser463/465), p-Smad2/3 (Ser465/467, Ser423/425), Smad 1, Smad5 and Smad2/3 was detected by Western blotting. (b) Representative gene expression of Collal, Colla2, Col3al, Ctgf, Fn1 and Acta2 was measured by qRT-PCR. (c) Protein expression of E-cadherin, $\alpha$-SMA, BMP-7, p-Smad1/5 (Ser463/465), Smad 1 and Smad5 was detected by Western blotting. (d) Representative gene expression of Colla1, Colla2, Col3al, Ctgf, Fnl and Acta2 was measured by qRT-PCR. (e) Protein expression of E-cadherin, $\alpha$-SMA, BMP-7, p-Smad1/5 (Ser463/465), Smad 1 and Smad5 was detected by Western blotting. All the experiments were repeated 3 times. ${ }^{*} p<0.05 ; * * p<0.01 ; * * p<0.001 ; \mathrm{NS}$, not significant.

ity and diabetic nephropathy, etc. ${ }^{26)}$ Among them, UUO is a simple and reproducible model which can reflect the characteristics of RIF in human obstructive nephropathy and chronic progressive kidney diseases. ${ }^{27)}$

Genistein, isorhamnetin, kaempferol and luteolin are four flavonoids in GbE. Among the four compounds, kaempferol has attracted our attention because of its broad-spectrum and strong inhibitory effect on the six fibrosis related genes. Therefore, we continued to study the anti-fibrotic effects of kaempferol in the subsequent experiments and tried to explore the mechanisms preliminarily. It was demonstrated that kaempferol not only ameliorated the EMT process of renal tubular epithelial cells induced by TGF- $\beta 1$, but also improved renal function decline and RIF in the UUO rats in a dosedependent manner.

TGF- $\beta 1$ has been identified as a key regulator of fibrosis and its role in RIF has been extensively studied. Therefore, TGF- $\beta 1$ is becoming an important target for many studies to design and develop new drugs that prevent RIF. In addition to the TGF- $\beta 1$ signaling pathway, we concentrated on another protein, BMP-7. BMP-7 is an anti-fibrotic protein and its antifibrotic effect is largely achieved by antagonizing the TGF- $\beta$ signaling pathway. For example, BMP-7 showed to inhibit the production of ECM in mesangial cells and retain the pheno- 

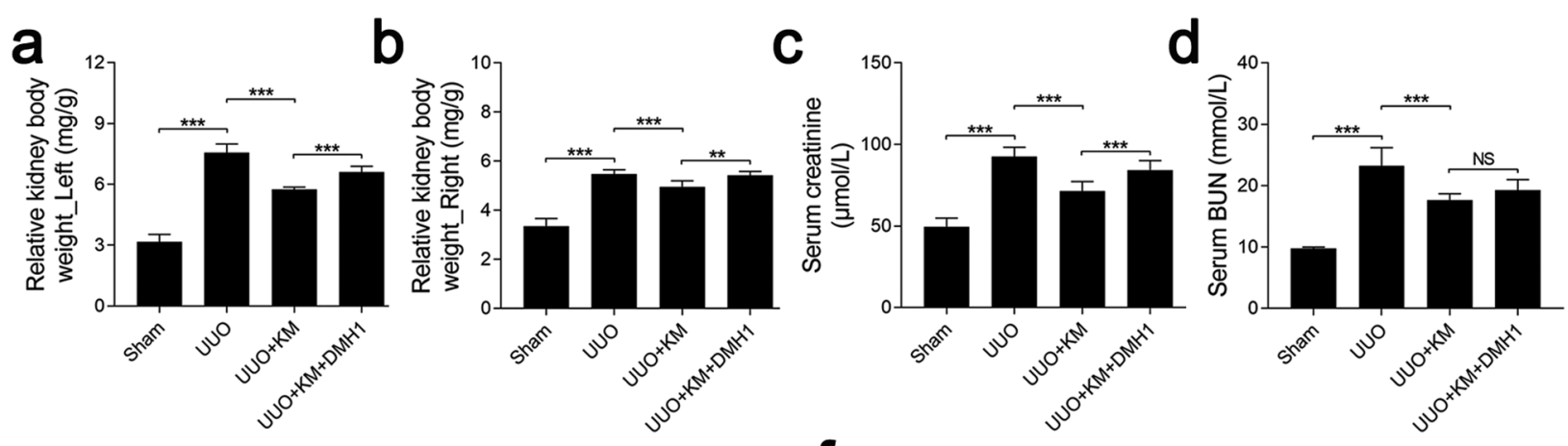

Q
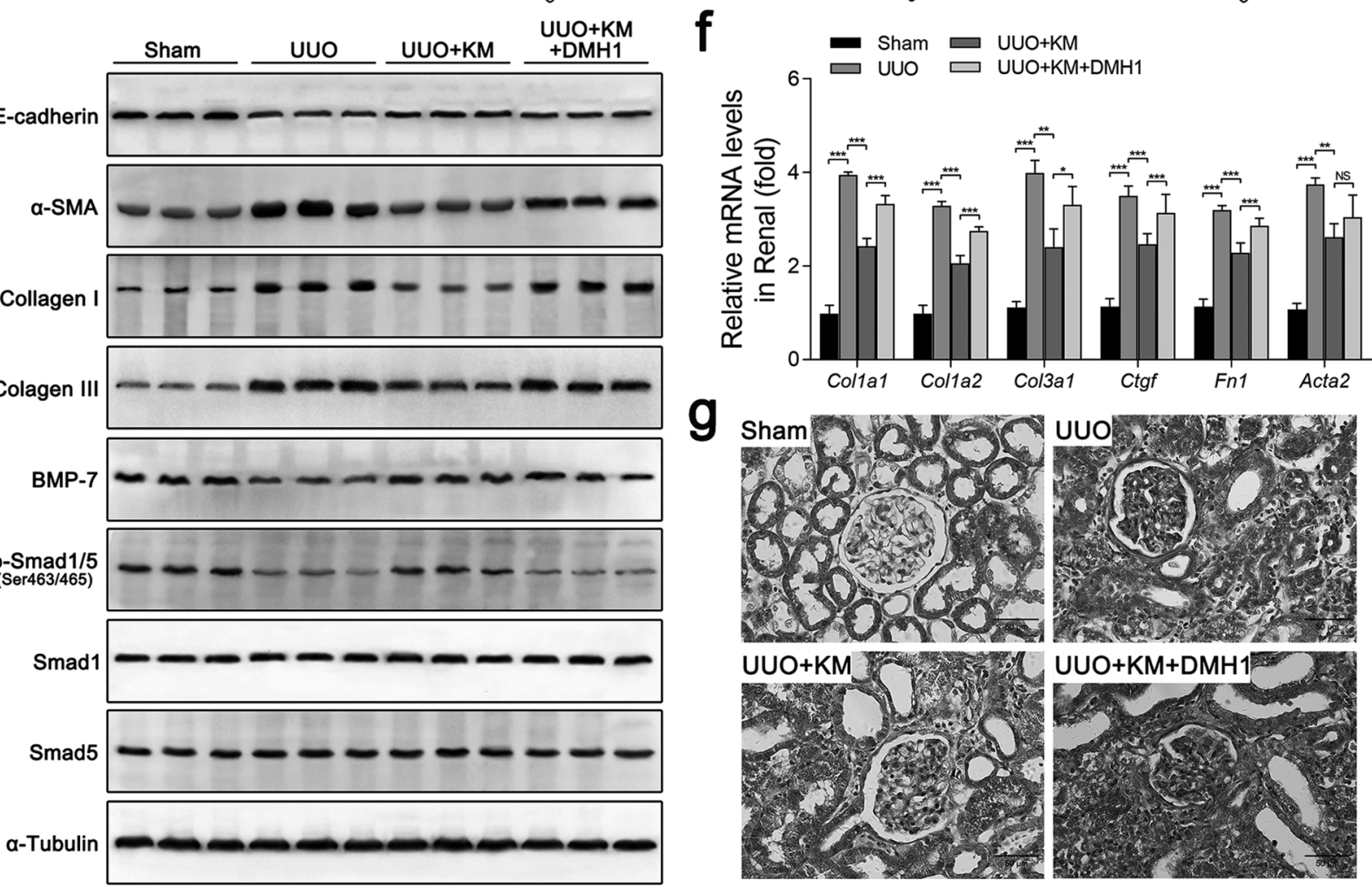

Fig. 4. Kaempferol Improves Renal Function Decline and Renal Interstitial Fibrosis in the UUO Rats through BMP-7-Smad1/5 Pathway

Relative weight of left (a) and right (b) kidneys to body weight of the rats was determined $(n=8)$. Serum creatinine (c) and BUN (d) levels of the rats were measured $(n=8)$. (e) Protein expression of E-cadherin, $\alpha$-SMA, Collagen I, Collagen III, BMP-7, p-Smad1/5 (Ser463/465), Smad 1 and Smad5 was detected by Western blotting $(n=4)$. (f) Representative gene expression of Collal, Colla2, Col3al, Ctgf, Fn1 and Acta2 in kidney of the rats was measured by qRT-PCR ( $n=4)$. (g) Masson staining of renal cortex sections of the rats in each group $(n=4)$. $*^{*} p<0.01 ; * * *<0.001$; NS, not significant.

type of renal tubular epithelial cells induced by TGF- $\beta 1{ }^{16,28)}$ To understand the mechanism by which kaempferol attenuates RIF, we examined the effects of kaempferol on TGF- $\beta 1$ and BMP-7 signaling pathways. Kaempferol treatment reversed the activity of Smad1/5, while had little effect on the phosphorylation levels of Smad2/3. These results indicated that the antifibrotic effects of kaempferol may be related to the activation of BMP-7-Smad1/5 signaling pathway. To further validate our hypothesis, DMH1 was utilized to inhibit the BMP-7-Smad1/5 signaling pathway in vitro and in vivo. As a result, effects of kaempferol on TGF- $\beta 1$ induced EMT in NRK-52E cells and UUO caused renal fibrosis in rats were greatly antagonized. Moreover, we found that kaempferol treatment obviously upregulated protein expression of BMP-7 in TGF- $\beta 1$ treated cells, which may be responsible for the activation of BMP-7-Smad1/5 signaling pathway. BMP-7 knockdown in NRK-52E cells further confirmed this hypothesis.

It is worth noting that DMH1 treatment or BMP-7 knockdown cannot completely reverse the improvement of renal fibrosis by kaempferol. We believe that the targets of natural compounds are extensive, and kaempferol is no exception. The BMP-7-Smad1/5 pathway is likely to be one of the targets of kaempferol. A recent study reported that kaempferol was able to attenuate renal fibrosis in diabetic nephropathy by inhibiting the RhoA/Rho-kinase. ${ }^{29)}$ In cardiovascular disease, kaempferol was reported to reduce vascular fibrosis induced by Ang II through the ERK1/2 and c-Jun N-terminal kinase (JNK) signaling pathways. ${ }^{30)}$ In this study, we focused on the effects of kaempferol on the two classic pathways related to renal fibrosis. Whether other molecules discussed above participate in the protection effects of kaempferol on renal fibrosis in UUO rats deserves further study.

In conclusion, we confirmed the strong potential of kaempferol in preventing RIF in vitro and in vivo. Through the initial exploration of the underlying mechanisms, we believed that the protective effects of kaempferol on RIF is closely related to the upregulation of BMP-7 protein and subsequent activation of Smad1/5 but not directly associated with the 
TGF- $\beta 1$-Smad2/3 pathway. However, the molecular mechanism of kaempferol on BMP-7 protein regulation was not elucidated and is worthy of further deep study. This research expands the boundary of kaempferol in disease treatment and provides a theoretical basis for the development of novel drugs for RIF therapy.

Acknowledgments This project was sponsored by the National Natural Science Fund of China (Nos. 81703100, 81673462, 81473293, 81403347, 81573604, and 91540119), the Fundamental Research Funds for the Central Universities. Six Talent Peaks Project in Jiangsu Province to Y.W. (YY-012). Key Research and Development Project of Jiangsu Province (BE2017712). The Science and Technology Development Foundation of Nanjing Medical University (2017NJMUZD065). Thanks to the Professor Xiaoxing Yin and Anzhou Xia from Xuzhou Medical University for giving NRK-52E cells and providing supports in animal breeding and modeling, respectively.

Conflict of Interest The authors declare no conflict of interest.

Supplementary Materials The online version of this article contains supplementary materials.

\section{REFERENCES}

1) Boor P, Ostendorf T, Floege J. Renal fibrosis: Novel insights into mechanisms and therapeutic targets. Nat. Rev. Nephrol., 6, 643-656 (2010).

2) Liu Y. Cellular and molecular mechanisms of renal fibrosis. Nat. Rev. Nephrol., 7, 684-696 (2011).

3) Loboda A, Sobczak M, Jozkowicz A, Dulak J. Tgf-beta1/smads and mir-21 in renal fibrosis and inflammation. Mediators Inflamm., 2016, 8319283 (2016).

4) Meng XM, Tang PM, Li J, Lan HY. Tgf-beta/smad signaling in renal fibrosis. Front. Physiol, 6, 82 (2015).

5) Lan HY. Diverse roles of tgf-beta/smads in renal fibrosis and inflammation. Int. J. Biol. Sci., 7, 1056-1067 (2011).

6) Sato M, Muragaki Y, Saika S, Roberts AB, Ooshima A. Targeted disruption of tgf-beta1/smad3 signaling protects against renal tubulointerstitial fibrosis induced by unilateral ureteral obstruction. $J$. Clin. Invest., 112, 1486-1494 (2003).

7) Rhyu DY, Yang Y, Ha H, Lee GT, Song JS, Uh ST, Lee HB. Role of reactive oxygen species in tgf-betal-induced mitogen-activated protein kinase activation and epithelial-mesenchymal transition in renal tubular epithelial cells. J. Am. Soc. Nephrol., 16, 667-675 (2005).

8) Hills CE, Squires PE. Tgf-betal-induced epithelial-to-mesenchymal transition and therapeutic intervention in diabetic nephropathy. $\mathrm{Am}$. J. Nephrol., 31, 68-74 (2010).

9) Carter NJ. Pirfenidone: in idiopathic pulmonary fibrosis. Drugs, $\mathbf{7 1}$ 1721-1732 (2011)

10) Sharma K, Ix JH, Mathew AV, Cho M, Pflueger A, Dunn SR, Fran$\cos$ B, Sharma S, Falkner B, McGowan TA, Donohue M, Ramachandrarao S, Xu R, Fervenza FC, Kopp JB. Pirfenidone for diabetic nephropathy. J. Am. Soc. Nephrol., 22, 1144-1151 (2011).

11) Meng XM, Chung AC, Lan HY. Role of the tgf-beta/bmp- $7 /$ smad pathways in renal diseases. Clin. Sci., 124, 243-254 (2013).

12) Wang SN, Lapage J, Hirschberg R. Loss of tubular bone morphogenetic protein-7 in diabetic nephropathy. J. Am. Soc. Nephrol., 12, 2392-2399 (2001). https://jasn.asnjournals.org/content/12/11/2392

13) Bramlage CP, Tampe B, Koziolek M, Maatouk I, Bevanda J, Bramlage $\mathrm{P}$, Ahrens $\mathrm{K}$, Lange $\mathrm{K}$, Schmid $\mathrm{H}$, Cohen $\mathrm{CD}$, Kretzler M,
Muller GA. Bone morphogenetic protein (bmp)-7 expression is decreased in human hypertensive nephrosclerosis. BMC Nephrol., 11, 31 (2010).

14) Hruska KA, Guo G, Wozniak M, Martin D, Miller S, Liapis H, Loveday K, Klahr S, Sampath TK, Morrissey J. Osteogenic protein-1 prevents renal fibrogenesis associated with ureteral obstruction. Am. J. Physiol. Renal Physiol., 279, F130-F143 (2000).

15) Wang S, Chen Q, Simon TC, Strebeck F, Chaudhary L, Morrissey J, Liapis H, Klahr S, Hruska KA. Bone morphogenic protein-7 (bmp-7), a novel therapy for diabetic nephropathy. Kidney Int., 63, 2037-2049 (2003).

16) Zeisberg M, Hanai J, Sugimoto H, Mammoto T, Charytan D, Strutz F, Kalluri R. Bmp-7 counteracts tgf-beta1-induced epithelial-tomesenchymal transition and reverses chronic renal injury. Nat. Med., 9, 964-968 (2003).

17) Zeisberg M, Shah AA, Kalluri R. Bone morphogenic protein-7 induces mesenchymal to epithelial transition in adult renal fibroblasts and facilitates regeneration of injured kidney. J. Biol. Chem., 280, 8094-8100 (2005).

18) Veerasamy M, Nguyen TQ, Motazed R, Pearson AL, Goldschmeding R, Dockrell ME. Differential regulation of e-cadherin and alpha-smooth muscle actin by bmp 7 in human renal proximal tubule epithelial cells and its implication in renal fibrosis. Am. J. Physiol. Renal Physiol., 297, F1238-F1248 (2009).

19) Lu Q, Zuo WZ, Ji XJ, Zhou YX, Liu YQ, Yao XQ, Zhou XY, Liu $\mathrm{YW}$, Zhang F, Yin XX. Ethanolic ginkgo biloba leaf extract prevents renal fibrosis through akt/mtor signaling in diabetic nephropathy. Phytomedicine, 22, 1071-1078 (2015).

20) Han Y, Lu JS, Xu Y, Zhang L, Hong BF. Rutin ameliorates renal fibrosis and proteinuria in 5/6-nephrectomized rats by anti-oxidation and inhibiting activation of tgfbetal-smad signaling. Int. J. Clin. Exp. Pathol., 8, 4725-4734 (2015).

21) Wang B, Liu D, Zhu QH, Li M, Chen H, Guo Y, Fan LP, Yue LS, Li LY, Zhao M. Rutin ameliorates kidney interstitial fibrosis in rats with obstructive nephropathy. Int. Immunopharmacol., 35, 77-84 $(2016)$.

22) Lu Q, Ji XJ, Zhou YX, Yao XQ, Liu YQ, Zhang F, Yin XX. Quercetin inhibits the mtorc1/p70s6k signaling-mediated renal tubular epithelial-mesenchymal transition and renal fibrosis in diabetic nephropathy. Pharmacol. Res., 99, 237-247 (2015).

23) Ozcan F, Ozmen A, Akkaya B, Aliciguzel Y, Aslan M. Beneficial effect of myricetin on renal functions in streptozotocin-induced diabetes. Clin. Exp. Med., 12, 265-272 (2012).

24) Cao J, Li Y, Peng Y, Zhang Y, Li H, Li R, Xia A. Febuxostat prevents renal interstitial fibrosis by the activation of bmp-7 signaling and inhibition of usag-1 expression in rats. Am. J. Nephrol., 42, 369-378 (2015)

25) Liu Y. New insights into epithelial-mesenchymal transition in kidney fibrosis. J. Am. Soc. Nephrol., 21, 212-222 (2010).

26) Nogueira A, Pires MJ, Oliveira PA. Pathophysiological mechanisms of renal fibrosis: a review of animal models and therapeutic strategies. In Vivo, 31, 1-22 (2017).

27) Chevalier RL, Forbes MS, Thornhill BA. Ureteral obstruction as a model of renal interstitial fibrosis and obstructive nephropathy. Kidney Int., 75, 1145-1152 (2009).

28) Wang S, Hirschberg R. Bmp7 antagonizes tgf-beta -dependent fibrogenesis in mesangial cells. Am. J. Physiol. Renal Physiol., 284, F1006-F1013 (2003)

29) Sharma D, Gondaliya P, Tiwari V, Kalia K. Kaempferol attenuates diabetic nephropathy by inhibiting rhoa/rho-kinase mediated inflammatory signalling. Biomed. Pharmacother., 109, 1610-1619 (2019).

30) Chen X, Zhang L, Wu G, Li H, Zhang F, Xu X. Kaempferol attenuates angiotesin ii-induced vascular fibrosis involving the jnk and ERK1/2 pathways. Int. J. Clin. Exp. Med, 9, 2407-2414 (2016). 